\title{
BMJ Open Comprehensive non-invasive prenatal screening for pregnancies with elevated risks of genetic disorders: protocol for a prospective, multicentre study
}

\author{
Chenming Xu, ${ }^{1}$ Xiaoqiang Cai, ${ }^{2}$ Songchang Chen, ${ }^{1}$ Qiong Luo, ${ }^{3}$ Hui Xi, ${ }^{4}$ \\ Dan Zhang (D) , ${ }^{5}$ Hua Wang, ${ }^{4}$ Yanting Wu (D) , ${ }^{1}$ He-Feng Huang, ${ }^{1,3}$ \\ Jinglan Zhang (i) ${ }^{1,2}$
}

To cite: Xu C, Cai X, Chen S, et al. Comprehensive noninvasive prenatal screening for pregnancies with elevated risks of genetic disorders: protocol for a prospective, multicentre study. BMJ Open 2021;11:e053617. doi:10.1136/ bmjopen-2021-053617

- Prepublication history and additional supplemental material for this paper are available online. To view these files, please visit the journal online (http://dx.doi.org/10.1136/ bmjopen-2021-053617).

CX, XC and SC contributed equally.

Received 19 May 2021 Accepted 13 August 2021

Check for updates

(c) Author(s) (or their employer(s)) 2021. Re-use permitted under CC BY-NC. No commercial re-use. See rights and permissions. Published by BMJ.

For numbered affiliations see end of article.

Correspondence to Dr Jinglan Zhang; jinglanzhang@foxmail.com and Dr He-Feng Huang;

huanghefg@hotmail.com

\section{ABSTRACT}

Introduction Chromosomal abnormalities and monogenic disorders account for $\sim 15 \%-25 \%$ of recognisable birth defects. With limited treatment options, preconception and prenatal screening were developed to reduce the incidence of such disorders. Currently, non-invasive prenatal screening (NIPS) for common aneuploidies is implemented worldwide with superiority over conventional serum or sonographic screening approaches. However, the clinical validity for the screening of frequent chromosome segmental copy number variations and monogenic disorders still awaits to be proved.

Methods and analysis This study is a multicentre, prospective study. The participants were recruited from three tertiary hospitals in China starting from 10 April 2021. The study is expected to conclude before 10 October 2022. Pregnant women with abnormal prenatal screening results indicated for invasive prenatal diagnosis or those who decide to terminate their pregnancies due to abnormal ultrasound findings will be evaluated for enrolment. Cell-free DNA extracted from the maternal plasma will be used for an analytically validated comprehensive NIPS test developed by Beijing BioBiggen Technology Co. (Beijing, China). The diagnostic results from prenatal or postnatal specimens as well as the pregnancy outcome data will be collected to examine the clinical sensitivity, specificity, positive and negative predictive values of the test.

Ethics and dissemination This study was approved by the Obstetrics and Gynecology Hospital of Fudan University (2020-178). Results of this study will be disseminated to public through scientific conferences and a peer-reviewed journal. Written informed consents will be obtained from participants.

Trial registration number ChiCTR2100045739.

\section{INTRODUCTION}

\section{Genetic aetiology of birth defects}

Birth defects are congenital conditions causing structural or functional anomalies at birth, which greatly contribute to infant mortality and disability. ${ }^{1}$ Approximately $3 \%-5 \%$ of newborns are affected by a birth
Strengths and limitations of this study

- This is the first prospective, multicentre clinical study for an integrated non-invasive prenatal screening test for both chromosomal abnormalities and monogenic diseases.

- This study is focused on a panel of preselected diseases which have relatively high incidence.

- The limitation for this study includes population stratification for high-risk pregnancies and potential loss of postnatal follow-up.

defect worldwide. ${ }^{2}$ Although the causes of most cases are unknown, about $15 \%-25 \%$ of birth defects are due to genetic diseases such as chromosomal abnormalities and monogenic disorders. ${ }^{3}$

\section{The screening of severe genetic diseases}

Great efforts have been made to prevent birth defects with an underlying genetic aetiology. Carrier screening for recessive disorders such as Tay-Sachs disease was proved to be highly effective for the reduction of its incidence. ${ }^{4}$ The first-trimester combined screening for fetal aneuploidies by prenatal ultrasound and maternal serum biochemical testing detects over $85 \%$ common trisomies at a false-positive rate of $\sim 5 \%$ which can lead to parental anxiety and excessive invasive diagnostic procedures for otherwise normal pregnancies imposing a risk for pregnancy loss. ${ }^{56}$ Since the discovery of circulating fetal cfDNA in the maternal plasma during pregnancy, its biological characteristics and clinical implication have been extensively studied. ${ }^{78}$ Noninvasive evaluation for fetal gender and risks for monogenic disorders, aneuploidies, and chromosome segmental CNVs were developed using different molecular or genomic techniques. ${ }^{9-11}$ Importantly, the emergence 
of next-generation sequencing (NGS) technology enabled a practical population-based screening method for Down syndrome. ${ }^{12} 13$ In the past decade, NGS-based non-invasive prenatal screening (NIPS) for trisomy 21, 18 and 13 has become a new standard for prenatal care with proven clinical validity. ${ }^{14}$ Recently, NIPS was used to detect rare autosome trisomies, sex chromosome aneuploidies, and microdeletion or microduplication syndromes. ${ }^{15} 16$ However, monogenic disorders which represent another major cause of birth defects are beyond the scope of the current screening of chromosomal abnormalities, and the clinical validity for the screening of such diseases are yet to be demonstrated.

\section{The development of NIPS for monogenic disorders}

Previous studies have shown that the analysis of fetal cfDNA was useful to determine the inheritance of parental alleles associated with autosomal or sex-linked recessive monogenic diseases. ${ }^{17} 18$ Additionally, noninvasive prenatal testing was also accurate for the diagnosis or screening of the de novo or paternally inherited variants causing dominant diseases such as achondroplasia and Noonan spectrum disorders. ${ }^{19} 20$ These studies showed potential clinical utility for monogenic NIPS (NIPS-M) in pregnancies at moderate risks (eg, pregnancies with advanced paternal age or ultrasound soft markers). These tests could also be used for the screening of diseases which can only be discovered at late gestational ages (eg, skeletal disorders). Although the analytical accuracy of NIPS-M has been well demonstrated, such tests will not be widely accepted without further evidence-based clinical study. ${ }^{21}$ First, the clinical validity of NIPS-M has not been supported by large prospective studies. The follow-up studies of the pregnancies tested positive or negative should be evaluated by clinical examination or golden standard diagnostic tests. Additionally, although isolated cases have demonstrated the benefits of NIPS-M leading to early diagnosis and better prenatal/postnatal management, the benefits for the management of patients with different indications are yet to be explored by larger studies. ${ }^{20} 2223$ Third, the limitations of NIPS-M need to be evaluated.
Accurate genetic counselling is critical to the success of a prenatal screening test which should provide information regarding disease characteristics, natural history, penetrance, expressivity, genotype-phenotype correlation, etc. The benefits and risks of NIPS-M need to be carefully evaluated when patients are counselled based on current understanding of the diseases. ${ }^{24}$

This study is aimed to address above important issues with a focus on the clinical validation of an innovative NIPS for concurrent screening of common aneuploidies, CNVs and monogenic disorders. The potential benefits and the limitations of this screening test will also be explored based on the pregnancy outcome data.

\section{Aims}

1. To assess the clinical validity of a novel NIPS test for concurrent screening of seven common aneuploidies, nine microdeletion and microduplication syndromes (MMS) and 155 monogenic disorders (75 genes).

2. To evaluate the pregnancy outcome for the participants of this comprehensive NIPS.

\section{METHODS AND ANALYSIS \\ Study design}

This prospective cohort study aims to evaluate how a comprehensive NIPS test will reveal pregnancies at risks for both chromosomal and monogenic disorders. It is a prospective, multicentre study focused on pregnant women with indications for prenatal diagnosis, including fetal ultrasound markers, high risk results by maternal serum screening or routine aneuploidy NIPS. Pregnancies with elective abortion due to fetal structural abnormality will also be recruited. To assess the performance metrics of this NIPS test, cases will be followed up to compare the screening results with the prenatal or postnatal diagnostic test results including sequencing, chromosomal CNV testing and/or karyotyping. Clinical follow-up will be pursued regarding the pregnancy outcome up to 6 weeks after birth (figure 1).

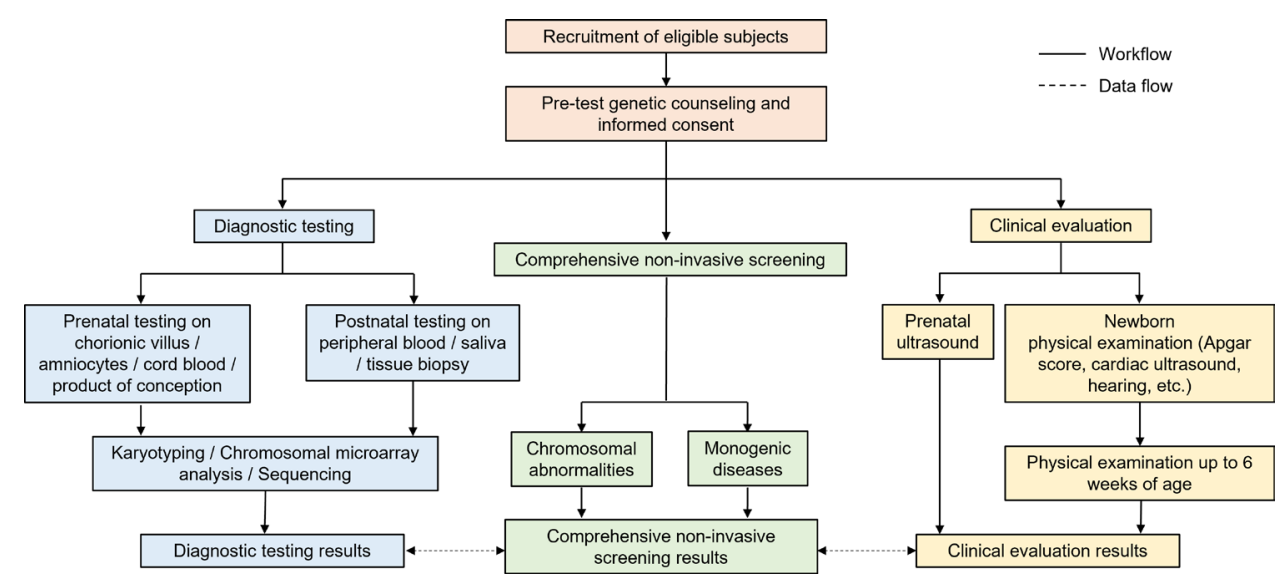

Figure 1 The diagram for the clinical validation of a comprehensive non-invasive prenatal screening test. 
Table 1 Inclusion and exclusion criteria

\begin{tabular}{|c|c|}
\hline Inclusion criteria & Exclusion criteria \\
\hline 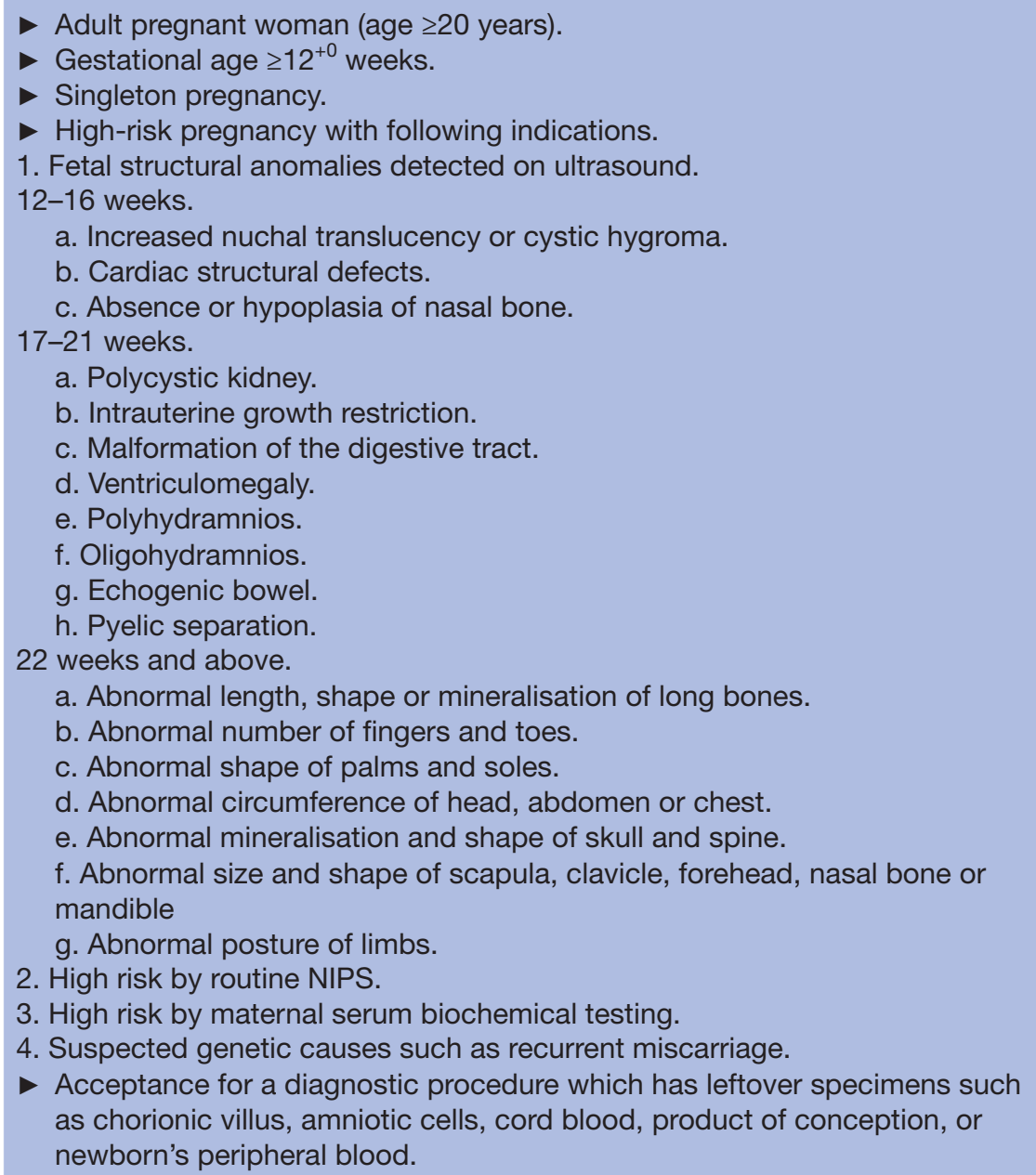 & $\begin{array}{l}\text { Age }<20 \text { years. } \\
\text { Gestational age }<12^{+0} \text { weeks. } \\
\text { Chromosomal abnormality in either of the } \\
\text { couple. } \\
\text { Received allogeneic blood transfusion, } \\
\text { organ transplantation, or cell therapy } \\
\text { within } 1 \text { year. } \\
\text { Family history of a genetic disease } \\
\text { indicated for an invasive diagnostic test. } \\
\text { Maternal malignancy during pregnancy. }\end{array}$ \\
\hline
\end{tabular}

\section{Consent and eligibility}

Participants will be recruited from three tertiary hospitals in China including the Obstetrics and Gynecology Hospital of Fudan University (Shanghai), the Hunan Provincial Maternal and Child Health Care Hospital (Changsha), and the Women's Hospital of Zhejiang University (Hangzhou). The detailed descriptions of inclusion and exclusion criteria are shown in table 1. Pretest genetic counselling will be provided by healthcare providers to all participants before obtaining the written informed consent to complete enrollment. The purpose and process of this study, as well as potential benefits, risks, data privacy and rights to withdraw will be discussed during the counselling session.

\section{Patient and public involvement}

Patients or the public were not involved in the design, conduct, or dissemination plans of this research.

\section{Sample size}

This study aims to recruit at least a total of 1000 pregnancies undergoing invasive diagnostic procedures or elective abortions due to abnormal prenatal findings suggestive of severe genetic disorders. All eligible subjects will be stratified into different indication groups (table 1), and the number of subjects in each group will be approximately allocated as the following: group 1, fetal structural anomalies detected by ultrasound ( $60 \%)$; group 2 , high risk by routine NIPS (20\%); group 3, high risk by maternal serum biochemical testing (10\%); group 4, suspected genetic causes with other indications (10\%). For group 1, further stratification will be achieved based on the gestational age, including 12-16 weeks, 17-21 weeks and 22 weeks and above (table 1). The number of subjects from each hospital will be evenly collected given equal participant availability. In this study, at least 1000 participants will be enrolled from whom we expect to detect at least 25 cases affected with a targeted monogenic or chromosomal disease each. This estimation is based on the detection rate among pregnancies with similar indications. ${ }^{25-27}$ The sample size in this study allows a probability of $95 \%$ or above to observe a possible measuring error at the case level for both the monogenic diseases and chromosomal diseases. 


\begin{tabular}{|c|c|}
\hline Type of diseases & Diseases and genes \\
\hline $\begin{array}{l}\text { Aneuploidies (7 } \\
\text { diseases) }\end{array}$ & $\begin{array}{l}\text { Trisomy } 21 \\
\text { Trisomy } 18 \\
\text { Trisomy } 13 \\
45, X \\
47, X X X \\
47, X X Y \\
47, X Y Y\end{array}$ \\
\hline $\begin{array}{l}\text { Microdeletion and } \\
\text { microduplication } \\
\text { syndromes ( } 9 \\
\text { diseases) }\end{array}$ & $\begin{array}{l}\text { DiGeorge syndrome } \\
1 \text { p36 deletion syndrome } \\
\text { 2q33.1 deletion syndrome } \\
\text { Angelman syndrome } \\
\text { Prader-Willi syndrome } \\
\text { Cri du Chat syndrome } \\
\text { Wolf-Hirschhorn syndrome } \\
\text { Langer-Giedion syndrome } \\
\text { Jacobsen syndrome }\end{array}$ \\
\hline $\begin{array}{l}\text { Monogenic } \\
\text { disorders (155 } \\
\text { diseases with } \\
\text { related } 75 \text { genes)* }\end{array}$ & $\begin{array}{l}\text { Representative diseases and genes: } \\
\text { Noonan spectrum disorders (PTPN11, } \\
\text { SOS1, RIT1, RAF1, etc.) } \\
\text { Osteogenesis imperfecta (COL1A1, } \\
\text { COL1A2, IFITM5) } \\
\text { Achondroplasia (FGFR3) } \\
\text { Crouzon syndrome (FGFR2, FGFR3) } \\
\text { CHARGE syndrome (CHD7) } \\
\text { Rett syndrome (MECP2) } \\
\text { Tuberous sclerosis (TSC1, TSC2) }\end{array}$ \\
\hline
\end{tabular}

*See supplemental materials for the full list of monogenic disorders.

\section{Screening and reporting}

The comprehensive non-invasive prenatal screening test used in this study was developed by Beijing BioBiggen Technology Co. (Beijing, China). This test is based on liquid-phase target enrichment followed by high readdepth NGS which can concurrently detect a panel of preselected diseases consisted of 7 common chromosomal aneuploidies, $9 \mathrm{MMS}$, and 155 monogenic disorders (table 2 and online supplemental table S1). A total of $10 \mathrm{~mL}$ peripheral blood is collected from each participant and the plasma is separated through a standard two-step centrifugation process. Manufacturer protocols are used for cfDNA extraction (TIANGEN, China) and NGS library construction (Nanodigbio, China). Custom designed hybridisation probes are synthesised and used for target enrichment (Integrated DNA Technologies, USA). The final DNA library is sequenced at $2 \times 100$ paired-end mode on MGISEQ-2000 sequencer (MGI, China).

The pathogenicity for both chromosomal and monogenic variants is evaluated according to the American College of Medical Genetics guidelines. ${ }^{28} 29$ Only pathogenic and likely pathogenic variants are deemed positive and reportable to patients after diagnostic confirmation. For cases with chromosomal abnormalities identified in the NIPS test, karyotyping, chromosomal microarray analysis, and whole genome sequencing for copy number variation analysis are used as diagnostic tests. For cases with screening positive monogenic variants, Sanger sequencing is used as the confirmatory test. Post-test genetic counselling is provided to participants by experienced clinical geneticists regarding the interpretation of the diagnostic results, the implications of these positive findings, and potential management options.

\section{Pregnancy outcomes follow-up}

All screening and diagnostic testing results, clinical examination results and images, and other relevant information available to us will be collected in the participants' medical records and used for statistical analysis. All cases will be followed up for pregnancy outcome including elective abortion, miscarriage, stillbirth or live birth. Newborns will be followed up for birth records and clinical examination or diagnostic testing up to 6 weeks of age. Subjects with positive NIPS results who do not have diagnostic genetic test results are excluded from the cohort. Subjects with negative diagnostic testing results, normal results in newborn physical examination or a genetic aetiology established for diseases other than those included in our screening panel are considered as negative cases.

\section{Statistical analysis}

The diagnostic testing and pregnancy outcome results of all pregnancies will be used to compare with the results generated by our NIPS test. The outcome is the area under the receiver-operating characteristic (ROC) curve for detection of each type of abnormalities (aneuploidies, MMS, and monogenic disorders) by NIPS in high-risk pregnancies. The ROC curve is generated by computation of test sensitivity and specificity, and CIs are computed using the Clopper-Pearson method. Assay performance metrics will also be demonstrated by false positive rate, false negative rate, positive predictive value, and negative predictive value, according to each category of abnormalities (figure 2). Data will be analysed with respect to different groups of indications for high-risk pregnancies, as well as pregnancies at different maternal age or gestational age.

\section{Study conduct}

In this study, subjects' demographic and clinical exam data are collected from test requisition forms, hospital medical records and postnatal follow-up surveys. Site monitoring of source data is performed following the study monitoring plan. All patents' privacy information collected during the study will be kept strictly confidential. Study data will be held securely on paper or electronically at the Obstetrics and Gynecology Hospital of Fudan University. Data safety will be reviewed on a regular basis to identify any safety concerns or trends.

\section{ETHICS AND DISSEMINATION}

This study was approved by the Obstetrics and Gynecology Hospital of Fudan University (2020-178). Results 


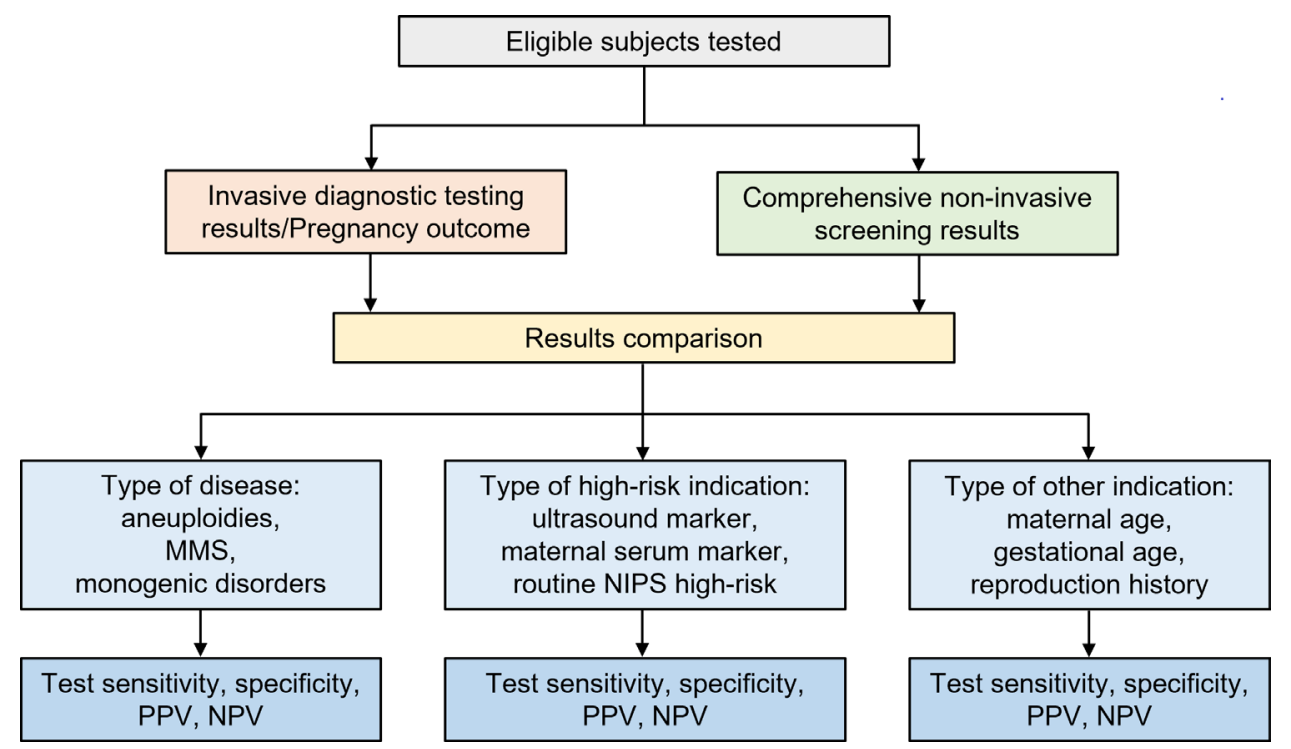

Figure 2 The diagram for the screening result analyses based on different disease types and indications. MMS, microdeletion and microduplication syndromes; NIPS, non-invasive prenatal screening; PPV, positive predictive value; NPV, negative predictive value.

of this study will be disseminated to public through scientific conferences and a peer-reviewed journal. Written informed consents will be obtained from participants. Deidentified participant data such as the screening and diagnostic results along with pregnancy outcome data will be shared at the individual or aggregative level. The assay protocols, clinical study protocol, statistical analysis plan, will be shared for at least 5 years after the clinical study is completed. The data will become available within 1 year after the clinical study is completed. All essential data supporting the conclusion of the study as well as detailed assay protocols, analytical algorithms, and customised computational codes will be submitted for publication within 1 year after the clinical study is completed. All the disease-causing variants and the key phenotypes found in the subjects will be published or deposited at a public database. Subjects' identifiable information including their genomic sequencing data will be kept in a clinical and privacy-compliant laboratory. Non-identifiable sequencing data (eg, individual variant data generated by locus-specific sequencing) can be provided on request from the corresponding author (Dr. Jinglan Zhang, jinglanzhang@foxmail.com) for at least 5 years after the clinical study is completed.

\section{DISCUSSION}

This is the first study to evaluate the clinical validity of an integrated non-invasive prenatal screening for both chromosomal abnormalities and monogenic disorders. Although NIPS for common aneuploidies has been accepted by professional societies and widely implemented around the world, NIPS for frequent chromosomal CNVs and monogenic disorders requires evidence-based investigation to prove its validity and its potential for pregnancy management. To investigate the performance of this new NIPS test, high-risk pregnancies are selected based on the routine prenatal screening results suggestive of genetic diseases and stratified into different groups. Diagnostic testing results together with pregnancy outcome data will be obtained for the clinical validation. Additionally, important practical issues around this test will be explored and discussed. For instances, the criteria for the selection of diseases, the proper indications for this test, genetic counselling and pregnancy management options will be evaluated based on the detection rate and pregnancy outcome to weigh the benefits and risks when offering a comprehensive NIPS test for different types of diseases. It should be noted that this study is observational, and all high-risk pregnancies recruited will be counselled by clinicians following current clinical guidelines for prenatal care. Patients' decisions regarding whether to take invasive diagnostic testing or how to proceed with their ongoing pregnancy will not be intervened by the screening results of this test unless it is confirmed by diagnostic testing. Previous studies (online supplemental table S2) assessing the clinical validity of aneuploidy or chromosomal CNV prenatal screening were also observational although most of these studies were conducted at an early gestational age when different pregnancy managing options are possible. ${ }^{14153031}$ The primary goal for this study is to assess the clinical validity of this new comprehensive NIPS test in a high-risk population in which abnormal prenatal screening results are mostly discovered at a late gestation age. Therefore, redirecting ongoing pregnancy can be challenging when the diagnostic test result is not available in time. Future study on general pregnancy population will be performed to investigate how this test may impact the prenatal or postnatal management at an early gestation age. 
Overall, in this prospective, multicentre study, we will provide invaluable data to assess the clinical validity of a novel comprehensive NIPS test for the concurrent screening of chromosomal and monogenic disorders. This test has the potential to be offered as an expanded and a next-generation NIPS test for general pregnancy population.

\section{Author affiliations}

${ }^{1}$ Obstetrics and Gynecology Hospital, Institute of Reproduction and Development, Fudan University, Shanghai, China

${ }^{2}$ Beijing BioBiggen Technology Co, Beijing, China

${ }^{3}$ Women's Hospital, School of Medicine, Zhejiang University, Hangzhou, Zhejiang, China

${ }^{4}$ Center of Hunan Provincial Prenatal Diagnosis, Hunan Maternal and Child Health Hospital, Changsha, Hunan, China

${ }^{5}$ Key Laboratory of Reproductive Genetics (Ministry of Education), Women's Hospital, Zhejiang University School of Medicine, Hangzhou, Zhejiang, China

Acknowledgements The technology development for this work was supported by Beijing BioBiggen Technology Co., Ltd.

Contributors JZ and H-FH conceived the study. JZ, H-FH, YW, CX and XC participated in the design of the study and drafting of the manuscript. CX, SC, QL, HX, DZ and HW participate in recruitment of participants and assessment of clinical outcomes. XC and JZ will design the statistical analysis plan and oversee statistical analysis. All authors critically reviewed and approved the manuscript for submission.

Funding This study was supported by The National Key Research and Development Program of China (2020YFA0804000) and the National Natural Science Foundation of China (82071661). The clinical validation and research work were partially supported by Technology Innovation Project of Shanghai Shenkang Hospital Development Center (SHDC12019107 and SHDC12018X17), the International Science and Technology Collaborative Fund of Shanghai (18410711800), the National Natural Science Foundation of China (81661128010), CAMS Innovation Fund for Medical Sciences (2019-12M-5-064), National Key Research and Development Program of China (2018YFC1002804), Program of Shanghai Academic Research Leader (20XD1424100), Outstanding Youth Medical Talents of Shanghai Rising Stars of Medical Talent Youth Development Program, Clinical Research Plan of Shanghai Shenkang Hospital Development Center (SHDC12018X17), and Clinical Research Project of Shanghai Municipal Health Commission (201840210, 20184Y0349). The cost of sample processing and highthroughput sequencing was supported by Beijing BioBiggen Technology Co., Ltd.

Competing interests XC, JZ are employees or shareholders of Beijing BioBiggen Technology Co. The other authors declare no conflict of interest.

Patient consent for publication Not required.

Ethics approval This study had been reviewed and approved by the Obstetrics and Gynecology Hospital of Fudan University (2020-178).

Provenance and peer review Not commissioned; externally peer reviewed.

Supplemental material This content has been supplied by the author(s). It has not been vetted by BMJ Publishing Group Limited (BMJ) and may not have been peer-reviewed. Any opinions or recommendations discussed are solely those of the author(s) and are not endorsed by BMJ. BMJ disclaims all liability and responsibility arising from any reliance placed on the content. Where the content includes any translated material, BMJ does not warrant the accuracy and reliability of the translations (including but not limited to local regulations, clinical guidelines, terminology, drug names and drug dosages), and is not responsible for any error and/or omissions arising from translation and adaptation or otherwise.

Open access This is an open access article distributed in accordance with the Creative Commons Attribution Non Commercial (CC BY-NC 4.0) license, which permits others to distribute, remix, adapt, build upon this work non-commercially, and license their derivative works on different terms, provided the original work is properly cited, appropriate credit is given, any changes made indicated, and the use is non-commercial. See: http://creativecommons.org/licenses/by-nc/4.0/.

\section{ORCID iDs}

Dan Zhang http://orcid.org/0000-0003-1295-4795
Yanting Wu http://orcid.org/0000-0002-2293-1792

Jinglan Zhang http://orcid.org/0000-0002-4600-7123

\section{REFERENCES}

1 Kancherla V, Oakley GP, Brent RL. Urgent global opportunities to prevent birth defects. Semin Fetal Neonatal Med 2014;19:153-60.

2 Mai CT, Isenburg JL, Canfield MA, et al. National population-based estimates for major birth defects, 2010-2014. Birth Defects Res 2019;111:1435:1420-35. doi:10.1002/bdr2.1589

3 Brent RL. Environmental causes of human congenital malformations: the pediatrician's role in dealing with these complex clinical problems caused by a multiplicity of environmental and genetic factors. Pediatrics 2004;113:957-68.

4 Antonarakis SE. Carrier screening for recessive disorders. Nat Rev Genet 2019;20:549-61. doi:10.1038/s41576-019-0134-2

5 Wapner R, Thom E, Simpson JL, et al. First-trimester screening for trisomies 21 and 18. N Engl J Med Overseas Ed 2003;349:1405-13. doi:10.1056/NEJMoa025273

6 Wright D, Syngelaki A, Bradbury I, et al. First-trimester screening for trisomies 21,18 and 13 by ultrasound and biochemical testing. Fetal Diagn Ther 2014;35:126 doi:10.1159/000357430

7 Jiang P, Lo YMD. The long and short of circulating cell-free DNA and the Ins and outs of molecular diagnostics. Trends in Genetics 2016;32:360-71. doi:10.1016/j.tig.2016.03.009

8 Lo YM, Corbetta N, Chamberlain PF, et al. Presence of fetal DNA in maternal plasma and serum. Lancet 1997;350:485-7. doi:10.1016/ S0140-6736(97)02174-0

9 Chiu RWK, Lau TK, Leung TN, et al. Prenatal exclusion of beta thalassaemia major by examination of maternal plasma. Lancet 2002;360:998-1000. doi:10.1016/S0140-6736(02)11086-5

10 Costa J-M, Benachi A, Gautier E. New strategy for prenatal diagnosis of X-linked disorders. N Engl J Med 2002;346:1502. doi:10.1056/ NEJM200205093461918

11 Lo YMD, Lun FMF, Chan KCA. Digital PCR for the molecular detection of fetal chromosomal aneuploidy. Proc Natl Acad Sci U S A 2007:104. doi:10.1073/pnas.0705765104

12 Chiu RWK, Chan KCA, Gao Y. Noninvasive prenatal diagnosis of fetal chromosomal aneuploidy by massively parallel genomic sequencing of DNA in maternal plasma. Proc Natl Acad Sci U S A 2008;105:20458-63.

13 Fan HC, Blumenfeld YJ, Chitkara U. Noninvasive diagnosis of fetal aneuploidy by shotgun sequencing DNA from maternal blood. Proc Natl Acad Sci U S A 2008;105:16266-71.

14 Norton ME, Jacobsson B, Swamy GK, et al. Cell-free DNA analysis for noninvasive examination of trisomy. $N$ Engl J Med 2015;372:1589-97. doi:10.1056/NEJMoa1407349

15 Liang D, Cram DS, Tan H, et al. Clinical utility of noninvasive prenatal screening for expanded chromosome disease syndromes. Genet Med 2019;21:1998-2006. doi:10.1038/s41436-019-0467-4

16 Srinivasan A, Bianchi DW, Huang $\mathrm{H}$, et al. Noninvasive detection of fetal subchromosome abnormalities via deep sequencing of maternal plasma. Am J Hum Genet 2013;92:167-76. doi:10.1016/j. ajhg.2012.12.006

17 Hudecova I, Jiang P, Davies J. Noninvasive detection of F8 int22hrelated inversions and sequence variants in maternal plasma of hemophilia carriers. Blood 2017;130:340-7.

18 Hui WWI, Jiang P, Tong YK, et al. Universal haplotype-based noninvasive prenatal testing for single gene diseases. Clin Chem 2017;63:513-24. doi:10.1373/clinchem.2016.268375

19 Chitty LS, Mason S, Barrett AN. Non-Invasive prenatal diagnosis of achondroplasia and thanatophoric dysplasia: next-generation sequencing allows for a safer, more accurate, and comprehensive approach. Prenat Diagn 2015;35:656-62.

20 Zhang J, Li J, Saucier JB. Non-Invasive prenatal sequencing for multiple Mendelian monogenic disorders using circulating cell-free fetal DNA. Nat Med 2019;25. doi:10.1038/s41591-018-0334-X

21 Scotchman E, Chandler NJ, Mellis R, et al. Noninvasive prenatal diagnosis of single-gene diseases: the next frontier. Clin Chem 2020:66:53-60. doi:10.1373/clinchem.2019.304238

22 Yan H, Zhu X, Chen J, et al. Noninvasive prenatal sequencing for multiple Mendelian monogenic disorders among fetuses with skeletal dysplasia or increased nuchal translucency. Prenat Diagn 2020;40:1459-65. doi:10.1002/pd.5792

23 Nwakalor C, Said-Delgado S, Krinshpun S, et al. De novo HRAS gene mutation associated with Costello syndrome identified by noninvasive cell-free fetal DNA screening. Prenat Diagn 2021;41:11-14. doi:10.1002/pd.5798 
24 Chitty LS, Hui L, Ghidini A, et al. In case you missed it: the prenatal diagnosis editors bring you the most significant advances of 2019. Prenat Diagn 2020;40:287-93. doi:10.1002/pd.5632

25 Norton ME, Brar H, Weiss J, et al. Non-Invasive chromosomal evaluation (NICE) study: results of a multicenter prospective cohort study for detection of fetal trisomy 21 and trisomy 18. Am J Obstet Gynecol 2012;207:137.e1-137.e8. doi:10.1016/j.ajog.2012.05.021

26 Lord J, McMullan DJ, Eberhardt RY, et al. Prenatal exome sequencing analysis in fetal structural anomalies detected by ultrasonography (page): a cohort study. Lancet 2019;393:747-57. doi:10.1016/S0140-6736(18)31940-8

27 Petrovski S, Aggarwal V, Giordano JL, et al. Whole-exome sequencing in the evaluation of fetal structural anomalies: a prospective cohort study. Lancet 2019;393:758-67. doi:10.1016/ S0140-6736(18)32042-7

28 Richards S, Aziz N, Bale S, et al. Standards and guidelines for the interpretation of sequence variants: a joint consensus recommendation of the American College of medical genetics and genomics and the association for molecular pathology. Genet Med 2015;17:405-23. doi:10.1038/gim.2015.30

29 Riggs ER, Andersen EF, Cherry AM, et al. Technical standards for the interpretation and reporting of constitutional copy-number variants: a joint consensus recommendation of the American College of medical genetics and genomics (ACMG) and the clinical genome resource (ClinGen). Genet Med 2020;22:245-57. doi:10.1038/s41436-0190686-8

30 Bianchi DW, Parker RL, Wentworth J, et al. Dna sequencing versus standard prenatal aneuploidy screening. N Engl J Med 2014;370:799-808. doi:10.1056/NEJMoa1311037

31 Lau TK, Chan MK, Lo PSS, et al. Clinical utility of noninvasive fetal trisomy (NIFTY) test--early experience. J Matern Fetal Neonatal Med 2012;25:1856-9. doi:10.3109/14767058.2012.678442 